\title{
The application of modified thermodynamic system correction method to secondary reheat steam turbine
}

\author{
Tongyang Pan ${ }^{1, *}$, Jifan Zhang ${ }^{1}$, Jiannan Kang ${ }^{1}$, and Mingcheng $\mathrm{Li}^{2}$ \\ ${ }^{1}$ Datang Northeast Electric Power Test \& Research Institute Co., Ltd., Changchun 130012, China \\ ${ }^{2}$ Datang Dongying Power Generation Co., Ltd., Dongying 257200, China
}

\begin{abstract}
In order to calculation the enthalpy of wet steam in the secondary reheat turbine thermal system the thermodynamic system of the secondary reheat steam turbine based on the isentropic ideal expansion process line was corrected, This method simplifies the correction calculation step and increases the accuracy of the correction result. Analysis of the rationality of the improved method shows that: Compared with the existing secondary reheat steam turbine thermal system correction method, the maximum error of the improved thermal system correction method is $0.14 \%$. Therefore, this method can better meet the test accuracy requirements.
\end{abstract}

Keywords: Secondary reheat steam, Thermodynamic system, Ideal enthalpy drop, Extraction pressure.

\section{Introduction}

When carrying out steam turbine thermal experiments, the operating parameters of the unit often have a large deviation from the design value of the unit and have a greater impact on the economics of the unit. Therefore, when the nature of the test is to verify the design and manufacture effect of the steam turbine, and to evaluate and diagnose the economic state of the turbine in the thermal system of the steam turbine to determine the heat rate of the turbine, the turbine body must be calculated by calculating the test value. The other conditions are corrected to the conditions specified in the design, and the error is reduced, that is, the thermal system correction [1-3].

In the current steam turbine test standard, the thermal system correction process is carried out under the condition that the efficiency of the flow passage portion of the steam turbine remains unchanged, that is, the actual expansion process line of steam in the steam turbine remains constant [4-5].Although In the literature [6] improved the current thermal system correction method, it still uses the correction calculation based on the actual steam expansion process line obtained during the test process. Although the literature [7] has revised the turbine thermal expansion process line, it is still based on the thermal process line obtained from the test conditions. In the literature [8], the steam turbine thermal correction method based on the isentropic ideal expansion process line is given, but the application of the secondary reheat unit is not given. 
It can be seen from the above analysis that the current secondary reheat steam turbine thermal system correction method is based on the actual steam expansion line obtained during the test process and remains constant during the correction process. However, in practical applications, the steam enthalpy and the low-pressure cylinder exhaust steam of the steam turbine in the wet steam zone required for the correction calculation can only be estimated because the humidity in the steam cannot be determined, so the actual expansion process line of the steam turbine itself There is a large error, which leads to inaccurate results of the various corrections of the thermal system and subsequent thermal economic evaluation and diagnosis. Therefore, this paper proposes an improved method for the existing thermal system correction method, which is mainly based on the ideal expansion process line of steam in the isentropic expansion of the steam turbine to modify the thermal system, effectively avoiding the steam in the wet steam zone. Depreciate the problem and make the correction result more accurate.

\section{Major items and contents of the thermal system correction}

In order to meet the thermal economy of the thermal system of the steam turbine unit and the accurate evaluation of the design and manufacturing effect, it is necessary to correct the parameters obtained by the actual test to the values corresponding to the operating conditions of the thermal system specified by the design to eliminate the factors other than the turbine body

The main items and contents of the thermal system correction usually include [8]:(1) The operation mode of the steam turbine regenerative system is the same as that specified in the initial design;(2) the feed water flow rate of the last high pressure heater outlet is equal to the main steam flow rate of the test steam turbine;(3) The outlet water (or condensate) temperature of each regenerative heater is different from the saturated water temperature of the corresponding heater vapor pressure, and the difference is equal to the specified heater end difference;(4) The inlet pressure of each regenerative heater is lower than the pressure measured at the extraction port, and the difference is equal to the pressure drop of the specified extraction line;(5) The condensate temperature of the lowest pressure heater inlet is equal to the saturation temperature of the turbine exhaust pressure minus the specified degree of subcooling, plus the temperature rise caused by the heat added to the condensate according to the regulations;(6) No desuperheating water;(7) The high pressure cylinder exhaust pressure is kept constant;(8) When the deaerator is operated at constant pressure, the deaerator outlet water raft shall be equal to the saturated water enthalpy at the rated pressure of the deaerator;(9) The increase in condensate pumps and feed pumps must comply with the manufacturer's design regulations;(10) The water storage capacity of the condenser hot well and the deaerator water tank is unchanged;(11) The heat loss of the extraction pipe complies with the manufacturer's design regulations.

\section{Improved thermal system correction method}

In order to avoid the problem that the last stage of the steam turbine is in the wet steam zone, and the low pressure cylinder exhaust steam is difficult to obtain, the thermal system correction method of the data obtained by the steam turbine test is improved. The main idea of the improvement is that the whole correction calculation process is based on the ideal isentropic expansion process line of steam in the steam turbine. The regenerative extraction steam is determined by the intersection of the isentropic expansion process line and the corresponding extraction point. The regenerative steam extraction amount of each stage of 
the ideal expansion process is calculated by using the condensing steam turbine variable working condition theory to calculate the steam pressure before and after the group.

\subsection{Calculate the leakage of steam in each shaft seal and the amount of steam leakage of the door stem when the steam supply parameters of the shaft seal are designed}

In the first correction calculation, only the shaft seal steam supply parameters adopt the design parameters, the other shaft seals and the door rod parameters take the test values, and the shaft seal and the door stem leakage amount are recalculated.

Due to the leakage of the ultra-high pressure door rod and the high-pressure and medium-pressure door stems, the steam leakage of the main steam valve and the regulating steam valve of each cylinder occurs before entering the super high, high and medium pressure cylinders of the steam turbine, and the steam is in the steam turbine. The expansion process has nothing to do, only depends on the parameters of the main steam and the first and second reheat steam. When the main steam and the first and second reheat steam parameters are constant, the amount of steam leakage remains the same as the test value. However, when the isentropic expansion of the steam in the turbine, the state of the shaft seal leak will change. For the actual expansion process of steam in the steam turbine, the steam state point before the shaft seal is the intersection of the actual expansion process line and the steam pressure before the shaft seal. When the steam is in the isentropic expansion process in the steam turbine, the steam state point before the shaft seal is the intersection of the isentropic expansion process line and the steam pressure line before the shaft seal.The calculation formula of the leakage of the shaft seal during the isentropic expansion of the steam turbine is:

$$
\Delta G_{1 \mathrm{t}}=\mu_{1} A_{1} \sqrt{\frac{P_{10}}{(Z+1.25) v_{10 \mathrm{t}}}}
$$

The calculation formula of the leakage of the shaft seal during the actual expansion of steam is:

$$
\Delta G_{1}=\mu_{1} A_{1} \sqrt{\frac{P_{10}}{(Z+1.25) v_{10}}}
$$

In the formula, $\Delta G_{1}$ and $\Delta G_{1 \mathrm{t}}$ are shaft leakage of the shaft seal for the expansion process, $\mathrm{kg} / \mathrm{s} ; \mu_{1}$ is the leakage of the shaft seal for the expansion process is the leakage coefficient of the shaft seal, according to the shaft seal tooth shape, the shaft seal tooth size and the shaft seal radial gap, by means of the shaft seal tooth profile flow coefficient curve diagram; $A_{1}$ is annular area of the shaft seal gap, $\mathrm{m}^{2} ; P_{10}$ is shaft seal front chamber pressure, $\mathrm{Pa} ; v_{10}$ and $v_{10 \mathrm{t}}$ are the steam specific volume of the front steam chamber for the two expansion processes, $\mathrm{m}^{2} / \mathrm{kg}$.

Comparing equations (1) and (2) can give the relationship between $\Delta G_{1}$ and $\Delta G_{1 \mathrm{t}}$ as:

$$
\Delta G_{1 t}=\Delta G_{1} \sqrt{\frac{v_{10}}{v_{10 \mathrm{t}}}}
$$


Thus, after knowing the actual amount of steam leakage of the shaft seal, the amount of steam leakage of the shaft seal during steam entropy expansion of the steam turbine can be calculated according to formula (3).

\subsection{Calculate the amount of regenerative steam extraction at all levels when the state of the regenerative system is intact}

When calculating the amount of regenerative steam extraction, the amount of leakage of the shaft seal and the door stem is calculated by using the shaft seal steam supply parameter as the design value. The regenerative extraction points are determined according to the measured regenerative extraction pressure and the isentropic expansion process line of the turbine. Since the water supply flow rate of the high-pressure heater system is known, at the same time, the corresponding parameters of the regenerative system and the relevant parameters of the heater are taken out, and the high-pressure level extraction amount can be obtained according to the heat balance relationship at the heater. The mass conservation is used at the deaerator to obtain the flow rate of the main condensate, so that the steam extraction amount of each low pressure stage can be further calculated.

\subsection{Correction of steam volume for small steam turbine of feed water pump}

During the actual operation of the steam turbine unit, the extraction point of the small steam turbine that supplies power to the feed water pump is located on the actual expansion process line of the steam. Therefore, in the thermal system correction method based on the isentropic expansion process, the ideal steam extraction amount should be calculated by converting the actual steam consumption of the small steam turbine to the isentropic expansion condition. Here, the actual steam consumption of the small steam turbine is converted into the current main water supply. The amount of steam extracted when the feed pump is pumped to the initial design value under flow.

The energy balance equation of the feed pump small steam turbine under actual expansion conditions is:

$$
G_{\mathrm{ep}} \Delta H_{\mathrm{tp}} \eta_{\text {rip }}=D_{\mathrm{fw}} \tau_{\mathrm{p}}
$$

In the ideal isentropic expansion process, the energy balance formula of the feed water pump is corresponding to the design value corresponding to the current main feed water flow:

$$
G_{\text {et }} \Delta H_{\text {tpt }} \eta_{\text {rip }}=D_{\text {fw }} \tau_{\text {pd }}
$$

In the formula, $G_{\mathrm{ep}}$ and $G_{\mathrm{et}}$ are the actual steam consumption of the feed water pump small steam turbine and the steam consumption of the isentropic expansion process, $\mathrm{kg} / \mathrm{s} ; \Delta H_{\mathrm{tp}}$ and $\Delta H_{\mathrm{tpt}}$ are the enthalpy of steam in a small steam turbine under different expansion process conditions, $\mathrm{kJ} / \mathrm{kg} ; \eta_{\text {rip }}$ is relative internal efficiency of feed pump small steam turbine; $\tau_{\mathrm{p}}$ is the feed pump in the actual operation is soaring, $\mathrm{kJ} / \mathrm{kg} ; \tau_{\mathrm{pd}}$ is corresponding to the initial design value of the feed pump soaring under the current main feed water flow rate, $\mathrm{kJ} / \mathrm{kg}$.

Comparing equations (4) and (5), the conversion formula for calculating the steam volume of the small steam turbine can be obtained: 


$$
G_{\mathrm{et}}=\frac{\Delta H_{\mathrm{tp}}}{\Delta H_{\mathrm{tpt}}} \frac{\tau_{\mathrm{pd}}}{\tau_{\mathrm{p}}}
$$

\subsection{Retrofit extraction steam pressure correction calculation}

The design value is obtained by the shaft sealing steam supply parameter, and the new shaft seal and the door stem leakage amount are obtained. The regenerative extraction amount is obtained by taking the state-prepared parameters through the regenerative system. At the same time, by using the feed pump to raise the design value, the steam consumption of the new feed pump small steam turbine is obtained. Therefore, under the condition that the design value of the shaft seal steam supply parameter is taken, the state of the heat recovery system is intact, and the feed water pump is upgraded to the design value, the steam flow rate of each stage group of the steam turbine flow passage portion is obtained. For the last stage group, since the pressure before the stage is proportional to the steam flow through the last unit, there is:

$$
P_{\text {es }}=P_{\text {etest }} \frac{G_{\mathrm{cs}}}{G_{\text {ctest }}}
$$

In the formula: $P_{\mathrm{es}}$ is the pressure before the final stage group after the system correction, MPa; $P_{\text {etest }}$ is the pressure before the last stage group under test conditions, MPa; $G_{\text {ctest }}$ is steam flow rate of the last stage group under test conditions, $\mathrm{kg} / \mathrm{s} ; G_{\mathrm{cs}}$ is the steam flow of the last stage group after the system correction, $\mathrm{kg} / \mathrm{s}$.

For each intermediate group, the formula is used to calculate the steam turbine variable working condition theory:

$$
\frac{G_{\mathrm{s}}}{G_{\text {test }}}=\sqrt{\frac{\left(P_{0}\right)_{\mathrm{es}}{ }^{2}-\left(P_{2}\right)_{\mathrm{es}}{ }^{2}}{\left(P_{0}\right)_{\text {test }}{ }^{2}-\left(P_{2}\right)_{\text {test }}{ }^{2}}}
$$

In the formula: $\left(P_{0}\right)_{\mathrm{es}}$ is the pressure before the middle group at the time of system correction, MPa; $\left(P_{2}\right)_{\text {es }}$ is the pressure after the middle group is corrected, MPa; $\left(P_{0}\right)_{\text {test }}$ is the pressure before the middle group at the test conditions, MPa; $\left(P_{2}\right)_{\text {test }}$ is the pressure after the middle group at the test condition, MPa; $G_{\text {test }}$ is the steam flow of the middle group at the test conditions, $\mathrm{kg} / \mathrm{s} ; G_{\mathrm{s}}$ is the steam flow of the middle group after the system is corrected, $\mathrm{kg} / \mathrm{s}$.

According to the above algorithm, each unit is calculated in turn from the last stage group, thereby obtaining the corrected front and rear pressures of the units of the medium and low pressure cylinders. Since the exhaust pressure of the high-pressure cylinder is kept the same as the test value, the high-pressure cylinders are still calculated by the Frugel formula, but the calculation is based on the high-pressure cylinder exhaust pressure, which is calculated from the back to the front. Corrected pressure of each hot recovery point of the high pressure cylinder. In the same way, the ultra-high pressure cylinder is also calculated based on its exhaust steam pressure.

Finally, we carry out the check of the regenerative extraction pressure. Comparing the regenerative extraction steam pressures obtained after the correction with the test values, if the relative change between the regenerative extraction steam pressures obtained after the 
correction calculation and the corresponding test values is less than $1 \%$, the obtained reheat extraction steam pressures are calculated. This is the final correction result. Otherwise, continue to calculate the new regenerative extraction steam pressure line and the ideal process line, determine the new regenerative extraction steaming point, determine whether the accuracy requirement is met, calculate the regenerative extraction amount and shaft seal leakage, and calculate the heat recovery. The amount of steam is extracted, and the regenerative extraction pressure is calculated until the pressure of each regenerative extraction point calculated by two adjacent calculations differs by $1 \%$..

\section{Improved thermal system correction method analysis}

The improved method of steam turbine thermal system proposed in this paper is to eliminate the influence of other factors other than the steam turbine body on the thermal economy of the unit without changing the efficiency of the steam flow in the cylinder of the steam turbine. That is to say, in the correction process, the efficiency of each unit and the relative internal efficiency of the turbine are guaranteed to be constant. Next, taking the simplified steam expansion process using the first-stage regenerative extraction as shown in Fig. 2 as an example, the thermal system correction process is analyzed to illustrate the rationality of the improved correction method.

In Fig. 1, number 1 point is the extraction state point of the actual expansion process measured by the test. number 2 point is the state points obtained by the existing system correction method based on the actual expansion line and corrected for 1 point. number 3 point is the state point of the isentropic expansion process line corresponding to number 1 point, number 4 point are the state points obtained by correcting number 3 point using the improved thermal system correction method.. Since the relative internal efficiency is kept constant during the correction process, the actual enthalpy and ideal enthalpy of the unit's extraction point have been increasing year by year regardless of the unit efficiency, that is, the rate of change of the points 1 and 3 is exactly the same.

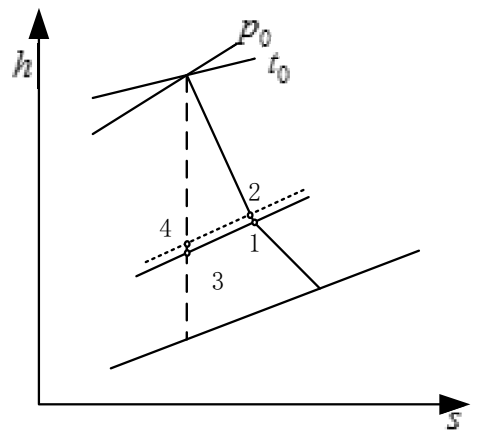

Fig. 1. Process line of System correction.

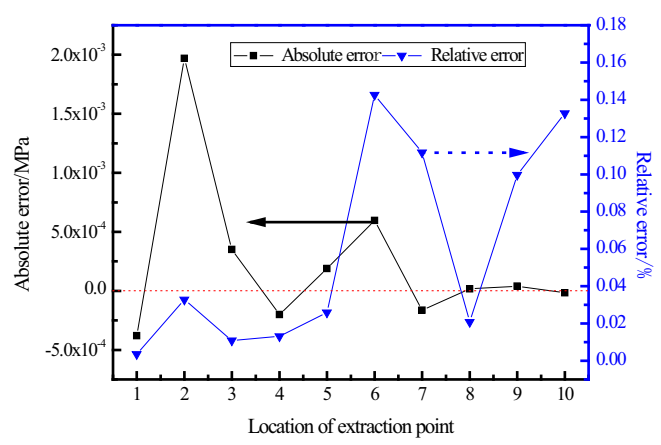

Fig. 2. Correction error.

Therefore, the correction results obtained by both the existing correction method based on the actual expansion process line of steam and the improved correction method based on the isentropic expansion process line are necessarily the same. This conclusion is shown in the figure as the extraction pressure obtained from the 1 point correction to 2 points, which is equal to the extraction pressure obtained from the 3 point correction to 4 points. The advantage of the improved thermal system correction method is that the solution process avoids the inaccuracy of the wet steam enthalpy, which simplifies the calculation steps and makes the error of the final correction result smaller and more accurate. 


\section{Improved correctness verification method for thermal system}

Table 1. Experimental value and revised value.

\begin{tabular}{|c|c|c|c|}
\hline \multirow[t]{2}{*}{ Extraction position } & \multirow{2}{*}{$\begin{array}{l}\text { Measured value of } \\
\text { extraction steam } \\
\text { pressure }\end{array}$} & \multicolumn{2}{|c|}{ Extraction steam pressure correction value (MPa) } \\
\hline & & $\begin{array}{c}\text { Actual expansion } \\
\text { process }\end{array}$ & $\begin{array}{l}\text { Isentropic expansion } \\
\text { process }\end{array}$ \\
\hline 1 & 10.817442 & 10.8313200 & 10.8317000 \\
\hline 2 & 5.997838 & 6.0023500 & 6.0003800 \\
\hline 3 & 3.242730 & 3.2172600 & 3.2169100 \\
\hline 4 & 1.518688 & 1.5286900 & 1.5288900 \\
\hline 5 & 0.731430 & 0.7337800 & 0.7335900 \\
\hline 6 & 0.414542 & 0.4186040 & 0.4180070 \\
\hline 7 & 0.146740 & 0.1467310 & 0.1468950 \\
\hline 8 & 0.085880 & 0.0855250 & 0.0855072 \\
\hline 9 & 0.03933 & 0.0392880 & 0.0392488 \\
\hline 10 & 0.012877 & 0.0122070 & 0.0122232 \\
\hline
\end{tabular}

The above analysis shows that the correction results calculated by the current thermal system correction method based on the actual steam expansion process line and the improved correction method based on the steam isentropic ideal expansion process line should theoretically be the same. In order to further illustrate the consistency of the results obtained by the two, the thermal system of a secondary reheat steam turbine is taken as an example to analyze the similarities and differences between the two correction methods.

Since the core problem of the correction calculation of the steam turbine thermal system is the correction of the pressure of the regenerative extraction point at each stage, only the correction result of the regenerative extraction pressure of each stage is calculated in this example. According to the actual thermal test data, the correction results based on the actual expansion process line and the isentropic ideal expansion process line are shown in Table. 1.

Comparing the correction results obtained by the two thermal system correction methods in Fig. 3, it can be seen that the absolute value of the absolute error of the two correction results is $0.00197 \mathrm{MPa}$, and the error analysis can be seen. In Figure 2, the maximum relative error is $0.1426169 \%$, and the error is extremely small, which fully meets the accuracy requirements of the thermal test in practical production applications.

\section{Conclusion}

The improved thermal system correction method considers the upper and lower ends of the regenerative heaters at each level and the pumping pressure loss of each pipeline, and based on the steam isentropic ideal expansion process line, recalculates the leakage flux of each shaft sealing rod under ideal conditions., the feed water pump small steam turbine leakage and regenerative steam extraction. The improved steam turbine thermal system correction method based on the isentropic ideal expansion process line, on the basis of ensuring the correctness of the test correction results, successfully avoids the wet steam enthalpy of the last few stages of regenerative extraction and exhaust steam problem. Thereby, the steps of the correction calculation are simplified, the calculation error caused by the inaccuracy of the wet steam enthalpy is reduced, and the accuracy of the correction result is improved. Taking each extraction pressure as an example, the absolute value of the absolute error of the correction result is at most $0.00197 \mathrm{MPa}$. Compared with the existing secondary reheat turbine thermal system correction method, the maximum error of the improved thermal system correction method is $0.14 \%$, to meet the accuracy requirements. 


\section{References}

1. Xi'an Thermal Power Research Institute. Steam turbine thermal test. Beijing: Electric Power Industry Press, 1982.158 162.

2. Liu Kai. Steam turbine thermal test. Beijing: China Electric Power Press, 2005.154 158.

3. Li Yong, Zhang Siwen, Li Hui. Research on thermal economy diagnosis method of steam turbine thermodynamic system based on improved equivalent enthalpy drop method. Steam Turbine Technology, 2009, 51(5): 333-337.

4. National Steam Turbine Standardization Technical Committee. GB8117-2008 Steam Turbine Thermal Performance Acceptance Test Regulations, Beijing: China Standard Press, 2009. 128 134.

5. ASME. ASME PTC 6-2004 Performance Test Codes 6 on Steam Turbine, New York: ASME Press, 2004. 58 59.

6. Zhang Caiwen, Huang Haizhou. Research on the Modification Method of Reheat Steam Turbine Performance Test System. Thermal Energy and Power Engineering, 2003, 18(3): 267-269.

7. Yao Yufeng, Wang Xinlei, Chen Dezhen. Modification of the unit matrix algorithm in calculating the thermal system of thermal power plants. Electricity and Energy, 2008, (5): 249-252.

8. Jiang Tieliu, Li Yong, Wang Jiayong, et al. Research on the improved method of steam turbine thermal system correction. Steam Turbine Technology, 2012, 54(04): 247-249. 